\title{
Nouvelle approche à la tâche de traduction du chevalier de Mailly
}

\author{
New approach to the translation work \\ by the chevalier de Mailly
}

\author{
Marina Pedrol-Aguilà \\ Universitat de Lleida \\ marina.pedrol@udl.cat
}

\begin{abstract}
Resumen
El caballero de Mailly (1657-1724) publicó una veintena de obras a lo largo de su carrera literaria, tres de las cuales fueron presentadas como traducciones: La Vie d'Adam, que provendría de un texto italiano; Histoire du prince Erastus, en la cual algunos críticos vieron la traducción de una novela en lengua italiana, otros la de una obra en español; por último, Le Voyage et les avantures des trois princes de Sarendip, que pretendía proceder de un texto en lengua persa. Mailly también dio a la imprenta un relato breve, "Avantures de Dom Leandre", cuyos primeros párrafos traducen el íncipit de una novela española. Además de intentar esclarecer la verdadera ascendencia de los originales que sirvieron de referencia a Mailly, queremos mostrar cómo evoluciona su concepción de la traducción literaria, pues comienza siendo muy fiel al texto fuente pero, poco a poco, se permite ciertas adaptaciones -ya sea en el fondo o en la forma- $y$, finalmente, termina adueñándose de la obra que traduce para crear una nueva.
\end{abstract}

\begin{abstract}
The chevalier de Mailly (1657-1724) published more than twenty works throughout his literary career, three of which were presented as translations. The aim of this paper is twofold. Firstly, to clarify the true origin of the works which Mailly translated: La Vie d'Adam, from a text originally in Italian; Histoire $d u$ prince Erastus, considered by some critics to be the translation of a novel written in Italian, by others the translation of a Spanish work, and finally, Le Voyage et les avantures des trois princes de Sarendip, which claimed to come from a text in Persian. Mailly also submitted for printing a tale, "Avantures de Dom Leandre", whose first few paragraphs are a translation of the incipit of a Spanish novel. The second aim is to analyse how Mailly's conception of literary translation evolved. At the beginning, the author remains faithful to his source text. Little by little, he succumbs to some modifications -in either form or contents-. Eventually, the writer ends up transforming the work he is translating by adding new stories to it.
\end{abstract}


Palabras clave

siglo XVII, siglo XVIII, literatura francesa, literatura italiana, literatura española.

\section{Key-words}

$17^{\text {th }}$ century, $18^{\text {th }}$ century, French Literature, Italian Literature, Spanish Literature.

\section{Introduction}

Au long de sa carrière littéraire, le chevalier de Mailly (1657-1724) publia une vingtaine d'ouvrages, dont trois parurent comme des traductions: La Vie d'Adam, avec des reflexions, traduite de l'Italien de Loredano (1695); l'Histoire du prince Erastus, fils de Diocletien (1709), où la critique a vu un original tantôt en langue italienne tantôt en langue espagnole, et Le Voyage et les aventures des trois princes de Sarendip, traduits du Persan $(1719)^{1}$. En outre, quoique sans l'expliciter, Mailly plaça en incipit de sa nouvelle "Les Avantures de Dom Leandre", comprise dans Diverses Avantures de France et d'Espagne, une traduction du début du Diablo cojuelo de Vélez de Guevara. Ainsi, paraît-il que notre auteur connaissait parfaitement l'italien et l'espagnol. Le statut de ces deux langues en France à la fin du XVII ${ }^{\mathrm{e}}$ siècle était ambivalent et les théories de la traduction s'avéraient nombreuses et souvent opposées. Après avoir brièvement présenté un état de la question à ce propos, dans le but d'éclaircir la véritable origine des œuvres que Mailly aurait traduites, nous aborderons leur étude en cherchant à vérifier le degré de fidélité du traducteur au texte source. Cette analyse devrait nous permettre de comprendre l'évolution de la conception maillynienne de la traduction littéraire.

\section{Contextualisation de la tâche de traduction du chevalier de Mailly dans le cadre des théories traductologiques en France aux XVIIe et XVIIIe siècles}

L'espagnol, l'italien et le français se font à l'époque concurrence en tant que langues de culture; Claude Lancelot (1660: II) affirmait: “c'est aujourd'huy en quelque façon vn plus grand reproche à vne personne de la Cour de ne pas sçauoir l'Italien, que de ne sçauoir ny le Grec ny le Latin". L'influence italienne en France était évidente: d'une part, sur le plan politique, avec Catherine et Marie de Médicis, puis Mazarin; d'autre part, sur le plan socio-culturel, avec Francesco Procopio; ou artistique et littéraire, avec les Comédiens Italiens, des musiciens comme Lully et des courants comme le marinisme. Quant à l'espagnol, il était forcément représenté à la cour, puisque Anne et Marie-Thérèse d'Autriche avaient été toutes les deux infantes d'Espagne avant de devenir reines en épousant respectivement Louis XIII et Louis XVI. Puis, l'influence du roman, de la nouvelle et du théâtre du Siècle d'Or espagnol sur la littérature française du Grand Siècle est indéniable.

Comme il est su, après un long combat dont les débuts remontent à la Renaissance,

1 Nous avons décidé de maintenir l'orthographe des titres et des textes cités. 
la langue française triomphe comme langue d'érudition en Europe, notamment à partir du Siècle des Lumières. Or, le XVII ${ }^{e}$ siècle particulièrement vit éclater des jalousies entre trois langues “sœurs": le français, l'italien et l'espagnol (Argaud, 2009: § 1).

Pourquoi cette comparaison s'est-elle effectuée de préférence avec l'italien et l'espagnol? De Bovelles note qu' “[...] Il y a trois langues vulgaires: l'italienne, la française, l'espagnole, tout à fait proches de la langue de Rome" (De Bovelles, 1531: 76). La comparaison entre ces trois langues se justifie donc amplement d'autant plus que ce sont "[...] les trois langues modernes qui ont le plus de vogue dans le monde [...]" (Bouhours, 1671: 126), donc susceptibles d'entrer en concurrence pour la prééminence. (Argaud, 2009: § 7)

Dès le $\mathrm{XVI}^{\mathrm{e}}$ siècle, Henri Estienne et d'autres lettrés ont réfléchi à cette supposée supériorité d'une langue vernaculaire sur une autre, qui portait sur des aspects tels que "la gravité, la gentillesse, la grace, la richesse, la brièveté ou la concision, qui désignent majoritairement des qualités abstraites et morales [...] que l'on croyait pouvoir identifier dans les caractéristiques sonores ou phonétiques ou encore dans le lexique" (Argaud, 2009: § 7). Cet intérêt porté sur la hiérarchie des langues découle de trois motifs qui convergent au $\mathrm{XVI}^{\mathrm{e}}$ et au XVII ${ }^{e}$ siècle. Le premier, de nature religieuse, est lié à la quête de la langue adamique -celle que l'on pensait universellement partagée avant l'épisode de la tour de Babel-. La deuxième raison relève de "la croyance qu'une langue reflète le caractère de la nation qui la parle", d'où que l'on cherche à l'embellir et à la perfectionner. Enfin, les tensions politiques entre pays s'expriment à travers un sentiment selon lequel la suprématie d'une nation irait de pair avec la prééminence de sa langue (Argaud, 2009: § 2-3). Dans cette sorte de combat, la traduction est, selon Guillerm (1996: 37) et Dotoli (2004: 102), une arme ${ }^{2}$ parce que le grand volume de traductions d'ouvrages espagnols et italiens en France aurait pour but de revendiquer la supériorité de la langue française sur ses deux langues "sœurs".

Néanmoins, la traduction est aussi un système de mise en valeur de la littérature étrangère car le succès des ouvrages traduits incite à traduire davantage de textes du même auteur, du même genre et, en fin de comptes, de la même langue. Dans ce sens, la traduction est en effet une arme, mais celle-ci est, si l'on file la métaphore, à double tranchant: d'une part, elle enrichit la langue et la littérature cibles; d'autre part, elle diffuse des littératures étrangères dont les langues voulaient être dépassées et reconnaît indirectement leur mérite. On ne peut pas oublier que "[1]'italien est un moyen pour nourrir le français [...] qui, à partir des années 1660, accomplit son parcours d'anoblissement. La traduction de l'italien devient un élément

2 Guillerm (1996: 37) remarque que “[c]e sont d'abord les Auteurs de langues vulgaires que l'on se permet [...] 'd'accoutrer', et tout particulièrement les auteurs de romans. Dans ce domaine, la rivalité est non seulement permise, mais légitime, voire politiquement justifiée, et les traductions se font aisément instruments de démonstration des supériorités du français sur l'espagnol, ou de sa compétition possible avec l'italien". Dotoli (2004: 102) manifeste que "[l]a traduction est une conquête pour donner à la France et à sa langue la primauté" puisque "[1]a comparaison est toujours à l'avantage de la France". 
de culture et de formation, dans tous les domaines" (Dotoli, 2004: 115). De sorte que celle qui était devenue la langue de Molière devait beaucoup à la langue de Dante car, si le français finissait par s'imposer comme langue de culture en Europe, "l'italien aurait contribué énormément à ce processus, à côté du grec et du latin. Les traducteurs de l'italien en français [...] savent qu'ils participent de la construction d'un modèle de langue, d'un idéal et d'une nouvelle littérature" (Dotoli, 2004: 117). Justement, la traduction dans la période classique connait, comme le remarque Bury, “deux axes majeurs [...]: d'une part, la 'conquête' des domaines étrangers, en vue d'enrichir la langue nationale [...], d'autre part l'exercice formateur pour le style même de celui qui traduit" (Zuber, 1995: 496). C'est ainsi qu'“au XVII' siècle, la traduction est un genre à tous les effets, qui se lie à ce que de nos jours nous appelons la sociologie de la littérature, dans son acception la plus vaste, des œuvres religieuses aux œuvres scientifiques", et à cela s'ajoute que "[1] es pratiques traductrices sont à la mode" (Dotoli, 2004: 107-108). Cependant, les traducteurs se plaignent toujours du manque de reconnaissance que l'on accorde à leur travail: si l'ouvrage traduit fait un succès, le mérite est attribué à son auteur original, tandis que si c'est un échec, la faute est toujours au traducteur, qui n'a pas été assez habile ou talentueux.

La traductologie est un sujet très vaste et complexe, et il ne s'agit pas ici de retracer son histoire, mais il convient de rappeler que, à partir des années vingt du XVII ${ }^{\mathrm{e}}$ siècle, l'on constate un regain d'importance de la traduction et, plus particulièrement, du prestige d'Amyot, l'un des traducteurs les plus renommés de la Renaissance, dont les préceptes recommandaient la quête de la clarté, par le choix des mots mais aussi par l'introduction de gloses dans le texte (Zuber, 1995: 26, 28 et 30). Giry et Godeau, surtout, conviennent que la fidélité est la clé pour mener à terme une bonne traduction et que les modifications ne doivent être qu'exceptionnelles (Zuber, 1995: 50-51). En 1638-1639, Conrart regroupe autour de lui une école de traducteurs guidés par l'éloquence, dont l'influence perdure jusqu'en 1644. Dans les années 1640, la méthode est commune à la plupart des traducteurs: l'on est généralement plus scrupuleux qu'à la Renaissance en ce qui concerne la fidélité à l'original, sans pour autant s'asservir à la traduction littérale, qui demeure réservée aux textes sacrés (Zuber, 1995: 74 et 77). Après 1645 et jusqu'en 1652, les entreprises commencées ne cessent de prospérer -d'Ablancourt, Du Ryer, Giry, Baudoin, Claveret ou Vaugelas continuent de traduire à un rythme plutôt intense- et quelques jeunes plumes débutent ( $\mathrm{Zu}$ ber, 1995: 97-99).

Il y a également, au XVII ${ }^{\mathrm{e}}$ siècle, un autre courant, inspiré des idées de Dolet et d'Érasme, dont le chef de file est Perrot d'Ablancourt, qui prône des traductions très libres où il faut essentiellement adapter le texte source au goût du public cible, tout en revendiquant ses propres mérites de créateur (Zuber, 1995: 104-107). Un bon mot de Ménage donnerait à ces textes le nom de "belles infidèles" (Zuber, 1995: 195-196). Dans les années 1650, à l'opposé de cette école, surgit le cercle de Miramion qui rattache la figure du traducteur à celle 
du docte, dont l'objectif premier doit être la diffusion des connaissances, et qui critique la traduction trop libre car elle manquerait à l'honnêteté intellectuelle. Vers le milieu du siècle pointe aussi l'école janséniste, qui tente d'impulser la création de règles pour traduire, mais il est difficile de trouver un accord (Zuber, 1995: 106-118).

Bury signale que, en 1654, d'Ablancourt publie son Lucien avec des Remarques sur la traduction, où il dit préférer 1"“agrément" à la "fidélité", idées qui préfigurent le goût classique, et, en cette même année, Marolles fait paraître une réédition des CEuvres de Lucain, dont il venait de revoir la traduction, qu'il avait faite lui-même en 1623, et dont la préface critiquait l'ambition poétique d'un traducteur contemporain. Cette confrontation annonce celle qui se produit entre d'Ablancourt et Huet au début des années 1660 (Zuber, 1995: 499500). En 1680, Huet publie un ouvrage capital, De Interpretatione libri duo, où il propose une méthode à mi-chemin entre la traduction sensuelle et littérale : il faut d'abord que le sens du texte d'origine soit correctement rendu puis, si la nature de la langue source et de la langue cible le permettent, l'on peut employer les mêmes mots et, enfin, reproduire le style de l'auteur que l'on traduit (Vega, 1994: 35).

Le début du XVIII ${ }^{\mathrm{e}}$ siècle voit naître une nouvelle querelle dans le domaine de la traduction (Yllera, 1996: 144-145). En 1713, Houdar de La Motte publie une traduction extrêmement libre de l'Illiade, faite à partir de traductions latines puisqu'il ignorait le grec, adaptée au goût du temps par l'altération de nombreux passages et la suppression de beaucoup d'autres. Cette méthode était fréquente et assez acceptée, mais La Motte ajoute à son œuvre un Discours sur Homère et une ode à L'Ombre d'Homère, où il critique les coutumes barbares des héros antiques, tout en feignant avoir reçu du grand poète grec la mission d'épurer son texte pour le rendre plus poli. $\mathrm{M}^{\mathrm{me}}$ Dacier, qui avait traduit l'Illiade bien plus fidèlement, réfute la méthode de La Motte dans Des Causes de la corruption du goût (1714). Ce traducteur répond par ses Réflexions sur la critique (1715). Le débat s'échauffe et l'Académie invite Fénelon à intervenir pour trancher la polémique. Celui-ci, dans une Lettre à l'Académie publiée en 1716, essaye de concilier les Anciens et les Modernes, tout en penchant insensiblement pour les premiers.

Il est évident que la traduction du $\mathrm{XVII}^{\mathrm{e}}$ siècle est héritière de celle du $\mathrm{XVI}^{\mathrm{e}}$; or, "les traducteurs du siècle nouveau [...] ne se présentent plus comme les porteurs d'un savoir inconnu, mais comme les révélateurs d'une expression moderne" (Zuber, 1995: 160). Par ailleurs, dans le choix des méthodes de traduction, Bury constate une "double postulation constante, qui veut associer la référence au modèle et l'affirmation d'une autonomie d'écrivain" (Zuber, 1995: 496). Il faut nonobstant remarquer que, bien qu'aux XVI ${ }^{\mathrm{e}}$ et XVII siècles il ait été plus intense ${ }^{3}$, le débat autour de la fidélité au texte original et des limites de

3 Dans sa Nouvelle allégorique, Antoine de Furetière (1658 : 70-71) donne un aperçu des méthodes traductives à la mode pendant la première moitié du Grand Siècle. La transposition de la querelle érudite sur un champ de bataille semble très juste: "A la gauche combatoient les Traductions en grand nombre, \& divisées en plusieurs corps; dont le premier marchoit sous Ablancourt Capitaine Magnifique, qui leur avoit donné des habits neufs 
la liberté du traducteur se pose à toutes les époques (Zuber, 1995: 415). En outre, il faut souligner que le travail de traduction est perçu comme un labeur difficile et pénible, qui s'avère extrêmement ingrat car il n'est pas assez valorisé -notamment lorsqu'on traduit des ouvrages de langues modernes-(Yllera, 1991: 641-642).

C'est au milieu de ce foisonnement de théories et de pratiques traductologiques que le chevalier de Mailly fit paraître La Vie d'Adam, avec des reflexions, traduite de l'Italien de Loredano (1695); Diverses Avantures de France et d'Espagne, qui contient "Les Avantures de Dom Leandre"; l'Histoire du prince Erastus, fils de Diocletien (1709) et Le Voyage et les aventures des trois princes de Sarendip, traduits du Persan (1719).

\section{De L'Adamo (1640) de Loredano à La Vie d'Adam (1695)}

En 1695, La Vie d'Adam, avec des reflexions fut publiée anonymement -puis réimprimée l'année suivante et rééditée en 1711-avec le sous-titre traduite de l'Italien de Loredano. Il s'agissait en effet, et plus précisément, d'une traduction de la huitième édition, parue en 1666 à Venise chez le Valvasense, de L'Adamo de Gian Francesco Loredano ${ }^{4}$ (Mailly, 1695c: “Avertissement"). Le Mercure galant annonçait la sortie de La Vie d'Adam dans un article qui fait notamment l'éloge de l'ouvrage original, mais qui consacre aussi quelques mots de louange au traducteur dont le nom n'est pas indiqué.

faits à la mode, qu'il avoit taillez \& rognez à sa fantaisie. Quelques autres obeissoiẽnt aux Capitaines Gyri [sic], Vaugelas \& Charpentier, dont la sévérité avoit rendu les troupes moins licencieuses; de sorte que sans céder aux autres en dignité, elles les surpassoient en justesse. Les derniers marchoient sous les Capitaines Vigenere \& Baudoin qui pour les avoir voulu trop grossir \& lever à la hâte; avoient esté obligez d'y enrôller plusieurs Drilles, dont les habits étoient déchirez en beaucoup d'endroits". Dotoli (2004: 109) explique que les défenseurs de ces trois groupes sont, respectivement: "ceux qui embellissent le texte, à la Nicolas Perrot d'Ablancourt, les traducteurs qui suivent le texte mot à mot, à la Vaugelas, à la Louis Giry et à la François Charpentier, et les traducteurs libres à la Blaise de Vigenère et à la Jean Baudoin".

4 Ce noble vénitien qui vécut entre 1606 et 1661 était issu de la famille des Doges du même nom; il fut trésorier au château de Palma-Nuova, sénateur, puis provéditeur de Peschiera (Weiss, 1820a: 37). Fondateur de la société littéraire de l'Accademia degli Incogniti, il fut romancier, poète et traducteur, et on lui doit de nombreux ouvrages comme Glorie degli Incogniti, Gli scherzi geniali, Vita del cavalier G. B. Marino, Il cimiterio cioe epitaffi giocosi, Morte e ribellioni del Valestain, Dianea, Sei dubbi amorosi, Novelle amorose, L'Iliade giocosa, Vita di Alessandro III pontifice Romano, Vita di Adamo, Bizarrie accademiche, Istoria de're Lusignani, Vita di S. Giovanni, vescovo Traguriense et une riche correspondance avec des écrivains contemporains (Weiss, 1820a: 37-38).

L"'Avertissement" d'Edme Couterot parle de Loredano comme de 1'“un des plus excellens Auteurs de son siecle", dont les ouvrages au style très beau étaient remplis d'érudition (Mailly, 1695c: s. p.); or, Weiss (1820a: 37) n'hésite pas à le qualifier de "médiocre". D'après Dotoli et al. (2001: 275-276), le XVII" siècle vit paraitre la traduction en français de quatre autres ouvrages de Loredano : La Dianée à M. le mareschal de Schomberg (Paris, A. de Sommaville et A. Courbé, 1642); Les Caprices héroïques du Loredano [traduit par François de Grenaille] (Paris, A. Robinot, 1644); L'Amour en son throne, ou Les Nouvelles amoureuses du Loredan gentil-homme venitien (Paris, A. Robinot, 1646); Lettres de Loredano, noble vénitien, sur diverses matières de politique et autres importans sujets... traduites en françois [...] par le sieur de Veneroni [Jean Vigneron] (Paris, Vve Marbre-Cramoisi, 1695). 
Il n'y a point de matiere si sterile qu'un genie heureux ne puisse rendre feconde. Rien ne paroissoit moins propre à estre traité, que la Vie d'Adam, puisque l'Ecriture nous apprend fort peu de chose de ce qu'a fait ce premier de tous les hommes. Cependant, Loredano. Noble Venitien, \& l'un des plus excellens Auteurs de son siecle, n'a pas laissé de nous donner cette Vie en Italien. Il est vrai qu'il y a ajousté des Reflexions Morales, qui ne servent pas seulement à embellir son Histoire, mais qui nous la rendent aussi utile qu'elle nous doit estre venerable. S'il a avancé des choses qui ne se rencontrent pas dans les Saintes Ecritures, Il l'a fait sur ce qu'en ont rapporté les Anciens Rabbins, qui ont écrit sur les livres de Moyse. Cette Vie d'Adam vient d'estre traduite par un homme qui a pris grand soin de conserver dans sa copie toutes les graces de l'Original, en sorte que l'on peut dire de luy qu'il connoist tres bien les beautez de l'une \& de l'autre langue. (Donneau de Visé, 1695a: 308-310)

Le texte de Mailly demeure très fidèle à sa source -nous avons pu le vérifier par une lecture comparative- même si, parfois, pour "rendre sa traduction plus Françoise \& plus agreable", l'auteur "a été obligé d'adoucir certains endroits", la langue française étant "plus serieuse \& moins libre que l'Italienne" (Mailly, 1695c: s. p.). Loredano et, donc, le chevalier narrent les quatre premiers chapitres du livre de la Genèse, s'arrêtant particulièrement à la création de l'Homme et de la Femme, ainsi qu'à leur péché et à leur punition. Ils abordent ensuite la vie d'Adam et d'Ève sur terre et l'histoire de leur descendance. Loredano et Mailly développent notamment l'épisode de Caïn et Abel, puis la bonne entente entre Adam et son fils Seth, à qui le premier homme confie ses visions sur l'avenir de leur postérité (le Déluge, la naissance de la Vierge, la passion et la mort du Christ, la délivrance des âmes retenues dans les Limbes et l'institution de la Loi nouvelle).

Nous avons été surprise par le fait que Mailly n'ait pas introduit d'éléments nouveaux $^{5}$, ce qu'il fit dans d'autres traductions, comme nous le verrons par la suite. Or, ce qui nous étonne le plus c'est qu'il ait conservé le discours extrêmement misogyne de Loredano. Le chevalier, qui adressa plusieurs de ses ouvrages au Beau Sexe, n'avait pas pour coutume de mépriser les femmes dans ses textes; elles étaient au contraire particulièrement valorisées dans la société galante qu'il fréquentait. Cette fidélité stricte au livre de Loredano pourrait découler de la méthode traductive généralement prônée pour les ouvrages religieux, qui veillerait à rendre la forme aussi bien que le fond.

5 Storer (2011: 168) affirme que "le récit biblique est transformé en histoire galante" et justifie sa thèse en citant la première rencontre d'Adam et Ève. Celle-ci "paroissait avoir quinze ou seize ans, comme toutes les héroïnes de roman romanesques", critique Storer (2011: 168). Adam, continue-t-elle en recopiant les mots de Mailly, "la trouva si belle, que l'admirer et l'aimer ne fut en luy qu'une même chose. Elle avoit des charmes si particuliers sur son visage, que c'eût été un effet de stupidité plutôt que de prudence, de n'en pas souhaitter la possession" et il la salua comme "aimable partie de moi-même, os de mes os, âme de mon âme, que je chéris plus que moimême” (Mailly, 1695c: 52-55 et Storer, 2011: 168). Or, ce sont les mots de Loredano (1666: 24-26) lui-même que le chevalier a traduits : 'L'osservarla, l'ammirarla, e l'amarla fù in un medesimo tẽpo. Portava nel volto doti cosi singolari, che il non dedicarle à se stesso, sarebbe stato effetto più tosto di stupidità, che di prudenza. [...] O'parte più cara più amata di me medesimo. Ossa delle mie ossa. Carne celle mie carni. Anima della mia anima". En ce qui concerne l'âge d'Ève, "era vicina al quarto lustro propria della dõna per la generatione, e per l'amore" (Loredano, 1666: 25). 
Quelques mois après sa parution, l'ouvrage traduit par Mailly donna lieu à une épître critique anonyme qui fut publiée dans le Mercure galant de décembre 1695. Cette lettre adressée à l'abbé B... accuse Loredano d'avoir trop légèrement abordé l'histoire du premier homme ${ }^{6}$ :

\begin{abstract}
Ce Noble Venitien s'est joüé visiblement de son sujet, \& sans respecter la source sacrée d'où il l'avoit tiré, il n'a songé qu'à le farder des plus vives couleurs de son éloquence, $\&$ à l'embellir. [...] Il a fait parler Dieu, Adam, Eve, \& tout ce qu'il y a d'autres sujets de cette Piece, en la manière qu'il a cru qu'ils devoient ou pouvoient parler; mais il y a meslé encore plusieurs faits, \& quelques circonstances qui n'ont aucun fondement dans l'Histoire, \& qui doivent uniquement leur origine à son Inventeur. (Donneau de Visé, 1695b: 74 et 83-84)
\end{abstract}

Malgré tous les reproches adressés à l'ouvrage de Loredano, ce virulent critique ne consacre pas un mot à la traduction de Mailly. Il est fort possible qu'il omît de le faire car le texte du chevalier reste très proche de celui du Vénitien. Mailly n'a vraiment été que le traducteur de l'ouvrage et il a repris autant que possible les mots dont l'écrivain italien s'était servi. Citons, à titre d'exemple, le passage où le serpent vainc Ève par la ruse et non pas lors d'une "guerre ouverte", ou le bref éloge de la chasse (Mailly, 1695c: 127, 165-166).

De même, dans le numéro de novembre 1695 de l'Histoire des ouvrages des savans, Basnage de Beauval (1730: 139) signalait: "On a traduit en François la vie d'Adam de l'Italien de Loredani [sic]: vous trouverez la bien des visions \& bien des contes rabbiniques". L'expression "visions \& [...] contes rabbiniques" montre une certaine méfiance envers le contenu de l'ouvrage; les Rabbins ayant "écrit plusieurs traditions superstitieuses, qu'ils observent aussi scrupuleusement que la Loi de Moise" (Dictionnaire de Trévoux, 1740: V, s.v. "rabbin").

\title{
4. Du Diablo cojuelo (1707) de Vélez de Guevara aux "Avantures de Dom Leandre", dans Diverses Avantures de France et d'Espagne (1707)
}

L'incipit des “Avantures de Dom Leandre", la cinquième nouvelle des Diverses Avantures de France et d'Espagne, est une traduction de celui du Diablo cojuelo de Luis Vélez de Guevara. Ce roman en langue espagnole, paru à Madrid chez Alonso Pérez en 1641, inspira également une libre adaptation d'Alain-René Lesage intitulée Le Diable boîteux. Bien que les Diverses Avantures de France et d'Espagne fussent publiées, comme Le Diable boîteux, en 1707 , le privilège du premier texte date du 11 décembre 1706, tandis que celui du second ne fut donné que le 5 juin 1707. Mailly ne put en aucun cas s'inspirer de Lesage, ce qui prouve qu'il avait des connaissances d'espagnol.

6 L'auteur de cette lettre cite d'autres d'ouvrages -français, espagnols, grecs et latins- où l'on brode amplement, de manière plus ou moins coupable, sur la matière biblique qui en a fourni le sujet principal (Donneau de Visé, 1695b: 75-83). 
Foulché-Delbosc (1899: 200) semble avoir été le premier à repérer ce "court fragment de traduction française de l'œuvre de Luis Velez de Guevara". Dans son article, qui est extrêmement succinct et presque vide de contenu, il conclut que "le chevalier de Mailly s'est efforcé de rendre fidèlement le texte de Guevara dont Lesage s'écarta de parti pris" (Foulché-Delbosc, 1899: 203). En effet, le passage traduit par Mailly -qui commence à la première ligne du récit et s'achève quelques pages plus tard (Vélez de Guevara, 1641: 1 r.-2 v.; Mailly, 1707a: 189-193) - reste assez proche de l'original. Cependant, une lecture comparative permet de constater quelques différences entre les deux extraits -original et traduction-que nous présentons ci-dessous en parallèle afin de mieux les appréhender.

$\S \S 1$ Daban en Madrid, por los Sur la fin du mois de Juillet, comme il étoit onze fines de Iulio, las onze de la heures du soir à Madrid, \& qu'il n'y avoit pas de noche en punto, hora men- clair de Lune: c'étoit le tems de la puissance de guada para las calles y, por l'Amour, le terme de la déclaration des Amans \& le faltar la luna, juridicción y moment heureux où ils renouvelloient les protestatérmino redondo de todo re-tions de s'aimer jusqu'à la mort.

quiebro lechuzo y patarata de

la muerte ${ }^{7}$.

$\S \S 2$ El Prado boqueaua coches en Le Prado de Saint Jerôme invitoit les carosses à la la última jornada de su paseo derniere journée de ses promenades ; \& dans les y en los baños de Mançanares bains du Mançanarez, les nouveaux Adams \& les los Adanes y las Euas de la nouvelles Eves de la Cour, se frotant plûtôt avec du Corte, fregados más de la sable, que se lavant avec de l'eau, prenoient congé arena que limpios del agua, de la riviere, \& disoient aux assistans Ite Rio est, aldezían el 'Ite, rio es', lez vous en, il n'y a plus de riviere pour vous.

$\S \S 3$ quando don Cleofás Lean- Ce fut alors qu'arriva l'avanture extraordinaire de Dom dro Pérez Zambullo, hidalgo Cleofas-Leandre-Perez-Zamboüillo. C'étoit un de ces a quatro vientos, cauallero Gentil-hommes aux quatre quartiers de la Boussole; un uracán y encruzijada de apel- Cleofas parmi les Juifs, un Leandre parmi les Grecs, lidos, galán de nouiciado y un Perez parmi les Chrétiens, \& un Zamboüillo parmi estudiante de profesión, les Mores. Il se nommoit encore le Chevalier de l'Ouragant \& quelquefois le Chevalier de la Tempête, tant il faisoit de bruit avec ses titres de Noblesse. Enfin, il prenoit tant de qualitez \& descendoit de tant de Heros, qu'on en auroit pu faire une croisade.

Il ne faisoit que d'entrer dans son noviciat de galanterie pour adoucir les chagrins attachez à l'étude des Lettres.

7 Puisque ces passages contiennent déjà des termes en italique, nous adopterons le soulignage pour mettre en valeur les passages qui diffèrent entre le texte source et le texte cible. 
$\S \S 4$ con un broquel y una espada, Ce jeune Heros de l'Université s'étant armé ou plûaprendía a gato por el caual- tôt embarassé d'un Bouclier \& d'un Espadon à deux lete de un tejado, huyendo de tranchans, grimpoit comme un chat sur le penchant la justicia, que le venía a los d'un toît assez rude, afin d'esquiver la Justice qui le alcances por un estupro que poursuivoit pour une prétenduë galanterie. Car quoino lo auía comido ni bebido, qu'il n'y eut pas eu de part, \& qu'il n'eut ni bû ni que en el pleito de acreedores mangé dans cette espece de regal, la Justice vouloit de una donzcella al uso estaua que le pauvre licencié payât lui seul l'écot, sur lequel graduado en el lugar vein- tant de gens avoient le reveillon, \& pour lui donner tidoseno, pretendiendo que ses grades dans les formes, elle lui avoit assigné la el pobre licenciado escotasse ving-deuxiéme place au nombre des Amans afidez solo lo que tantos auían me- d'une de ces Demoiselles destinées à l'usage du purendado; y como solicitaua blic. Ce nouveau gradué cherchoit les moyens de se escaparse del 'para en uno retirer de cette intrigue embarassante à quelque prix son'-sentencia definitiua del que ce pût être. Il y avoit déja contre lui une Sentence cura de la parroquia y auto diffinitive [sic] du Curé de la Paroisse, \& un acte auque no lo reuoca si no es el thentique qui ne peut être revoqué que par le grand vicario Responso, Iuez de la Vicaire, Juge établi pour les affaires de l'autre vie. otra vida-,

$\S \S 5$ no dificultó arrojarse desde De sorte que se sentant par là aussi pressé qu'un el ala del susodicho tejado, homme qui a pris medecine, il ne fit pas difficulté de como si las tuniera a la buar- s'élançer d'une des aîles de ce toît fort élevé, fur le da de otro que estaua confi- trou d'un autre toît qui en étoit assez proche. Ce qui nante, nordesteado de una le détermina à prendre ce parti, fut une petite lumiere luz que por ella escasamente sombre qu'il crût lui devoir servir d'Etoile polaire, se brujuleaua, estrella de la ou d'Etoile du matin, mais en effet, c'étoit l'Etoile tormenta que corría, en cuyo de fa mauvaise fortune \& de la tempête qu'il alloit desuán puso los pies y la boca essuyer. Il se jetta donc avec tant d'impetuosité qu'il a un mismo tiempo, saludán- mit en même tems les pieds \& la tête fur le plancher dolo como a pue[r]to de tales du galetas, où il fit la culbute malgré toute fon agilinaufragios y dexando bur- té. Cependant il ne laissa pas de regarder cet endroit lados los ministros del agarro comme un port favorable qui le mettoit à couvert du y los honrados pensamientos naufrage. Aprés avoir repris ses sens, il mit la tête au de mi señora doña Tomasa de travers le trou par où il avoit tombé, \& se moqua des Bitigudiño, donzella chanflo- Officiers du Guet, \& des beaux desseins qu'avoit sur na que se passaua de noche lui Dame Tomaze de Bitigourligno, Demoiselle de la como quarto falso [...]. race des souffleurs; car la nuit elle ne laissoit pas que de passer comme font les faux quart-d'écus. 
Malgré la fidélité de la traduction du chevalier, nous ne pouvons point passer sous silence qu'il ajouta à l'incipit de Vélez de Guevara quelques éléments nouveaux. L'on constate plusieurs phénomènes d'amplificatio en ce qui concerne, notamment, la présentation du héros de la nouvelle. Mais l'on peut dire que Mailly brode sur l'ensemble de l'extrait car, quoiqu'il reprenne bien souvent les mots de l'écrivain espagnol, il rajoute plusieurs développements. D’une part, il cherche à mieux décrire les évènements narrés ( $\S 1,2$ ou 5$)$, ce qui pourrait être parfois lié aux effets du conceptisme espagnol, très difficile à traduire $(\S 1)$. D'autre part, le chevalier introduit par son amplificatio certains détails utiles à l'intrigue de sa nouvelle. Dom Leandre est, dès le début, caractérisé comme un personnage orgueilleux et fanfaron (§ 3) et un peu ridicule: rappelons qu'il est embarrassé de ses armes (§ 4) et que son urgence pour échapper au guet est comparée à celle de quelqu'un qui aurait pris un laxatif ( $\S$ 5). Mailly se servit donc des premières pages du roman de Vélez de Guevara, où sont présentés le cadre spatio-temporel et le personnage principal, pour établir les bases de son propre récit, qui ne garde par la suite aucun point commun avec El Diablo cojuelo, à l'exception du nom du héros.

\section{De l'anonyme Histoire pitoyable du prince Erastus, fils de Diocletien, Empereur de Rome (1564) à l'Histoire du prince Erastus, fils de l'empereur Diocletien (1709)}

La parution de ce titre de Mailly est annoncée dans le Mercure galant (Donneau de Visé, 1709: 187-198) du mois de décembre, parmi les "Livres nouveaux". Les auteurs du périodique consacrent à l'Histoire du prince Erastus un grand nombre de pages où sont repris, d'abord, les mots de l'“Avertissement” de Mailly (1709c) qui décrivent brièvement l'histoire des traductions de ce roman ${ }^{8}$. S'ensuit une présentation des personnages principaux de l'ouvrage et un abrégé du livre. Pour notre part, nous résumons aussi l'argument afin de faciliter la compréhension des explications postérieures.

Erastus, fils de l'empereur Dioclétien, est un jeune prince dont les parfaites qualités augurent un avenir prometteur. Il est éduqué, loin du tumulte de Rome, par sept philosophes qui lui apprennent les sept arts libéraux et l'art de la politique. Ayant perdu sa première femme, Dioclétien épouse Aphrodisia. Celle-ci entend parler merveilles d'Erastus et finit par convaincre l'empereur de le faire rappeler à la cour. Avant de partir, le prince consulte les astres et se voit annoncer une mort ignominieuse s'il rentre à Rome, mais aussi de grands honneurs s'il est capable d'observer le silence pendant sept jours. Dès qu'elle trouve l'occasion, Aphrodisia s'offre au prince mais celui-ci refuse ses avances. Dépitée, l'impératrice l'accuse d'avoir tenté de la forcer. Dioclétien, déconcerté par le silence de son fils -qu'il interprète comme un aveu de culpabilité- et pressé par sa femme, le condamne à mort. Com-

8 Le chevalier parle d'une version originale en grec, puis d'une traduction en italien, d'une autre en espagnol et, enfin, d'une traduction en vieux français (Mailly, 1709c: s. p). Il est curieux de signaler que l'article du Mercure galant (Donneau de Visé, 1709: 187-188) rajoute une traduction en latin. 
mence alors un procès très singulier pendant lequel les sept sages, à tour de rôle, et Aphrodisia narrent alternativement des histoires pour convaincre Dioclétien: les philosophes dénoncent systématiquement la malice des femmes et l'impératrice plaide contre l'ingratitude filiale ${ }^{9}$. Enfin libéré de son vœu de silence, Erastus s'explique et est innocenté. Aphrodisia est arrêtée et emprisonnée, mais l'horreur de son crime la pousse à se suicider.

L'Histoire du prince Erastus provient de la matière des Sept sages de Rome. Laufer (1971: 189) met l'accent sur le rattachement du conte-cadre du roman de Mailly au mythe de Phèdre et d'Hippolyte car, d'après lui, l'enchaînement de récits insérés n'est qu'un prétexte pour illustrer "tour à tour les points de vue de l'homme et de la femme". Il pense également que "le chevalier de Mailly n'avait fait que profiter de la vogue récente donnée à ce thème ${ }^{10}$ par l'Histoire de la sultane de Perse et des vizirs" (Laufer, 1971: 189), que Pétis de La Croix avait publiée en 1707 (Paris, veuve Barbin). En fait, ce récit est tiré du conte des "Sept vizirs", compris dans Les Mille et Une Nuits -traduites en français par Galland et dont le premier volume, paru en 1704, eut un énorme succès-.

Justement, l'histoire des Sept vizirs et celle des Sept sages de Rome proviennent de la même matière, qui est très complexe de par son ancienneté et de par le grand nombre de versions qui en ont surgi au fil du temps. À l'origine, il s'agissait d'une collection de contes orientaux $^{11}$, qui fut largement diffusée en Europe à partir du Moyen Âge. Cette transmission se fit en deux branches : l'une, orientale, connue sous le nom de Livre de Sindbād ou Sindban, et l'autre, occidentale, divulguée sous le titre de Livre des Sept sages de Rome ${ }^{12}$ (Runte et al, 1984: xiii). Il est important de souligner qu'en Occident, la tradition française des Sept sages est considérée la plus riche et la plus influente (Runte et al, 1984: 64). L'Histoire du prince Erastus appartient à la versio italica, un ensemble de textes de la branche occidentale qui partagent des caractéristiques communes en ce qui concerne la chronologie de la narration, le nombre de récits encadrés, ainsi que la position toujours initiale du conte appelé Canis ${ }^{13}$ (Hurtado de la Vera, 1996: 15).

Au milieu du XVIII ${ }^{\mathrm{e}}$ siècle, l'abbé Francesco Saverio Quadrio retrace l'histoire de ce texte depuis l'Antiquité. Quant à sa réception en France, d'après lui, ce roman aurait été translaté de l'italien au français par un auteur anonyme, puis imprimé à Lyon et à Anvers en

9 Le conte-cadre des Mille et Une Nuits, où Schéhérazade a recours à l'art de conter pour repousser sa propre exécution de jour en jour, relève de la même formule littéraire.

10 Bien que l'ouvrage de Pétis de La Croix soit bien plus proche -dans le temps et par rapport à la structure- de celui de Mailly, il ne faut pas oublier le grand référent que Racine avait créé en 1677 avec Phèdre et Hippolyte, dont le sujet central est étroitement lié à la matière développée dans l'Histoire du prince Erastus.

11 L'on ignore la provenance exacte du recueil (d'où qu'il soit qualifié d'oriental sans davantage de précisions), mais ce qui est certain c'est qu'il y eut une traduction arabe qui donna lieu aux versions européennes, qui dérivent plus ou moins directement de celle en langue arabe (Deyermond, 2006: 74).

12 La branche occidentale serait issue d'une traduction latine datant du XII ${ }^{\mathrm{e}}$ siècle, qui avait modifié le modèle original de la branche orientale (Gómez Redondo, 2006: 105).

13 C'est la terminologie latine établie par la critique au XIX ${ }^{\mathrm{e}}$ siècle qui est employée pour désigner les histoires insérées dans les romans inspirés de la matière des Sept sages (Runte, 2014). 
1568, à Paris en 1572 et en 1579 , et à Rouen en 1616 , "e poi di nuovo tradotto nel corrente secolo dal Cavalier di Mailli” (Quadrio, 1749: 453) ${ }^{14}$. L'original italien et l'anonyme français dont parle l'abbé seraient, respectivement: I compassionevoli avvenimenti d'Erasto, paru pour la première fois à Venise en 1542, et l'Histoire pitoyable du prince Erastus, fils de Diocletien, empereur de Rome, publié à Lyon en 1564 et rééditée, en effet, maintes fois au long des XVI ${ }^{\mathrm{e}}$ et XVII ${ }^{\mathrm{e}}$ siècles ${ }^{15}$.

Les bibliographes du XIX ${ }^{\mathrm{e}}$ siècle présentent le texte de Mailly comme une "traduction nouvelle", tantôt de l'espagnol (Weiss, 1820b: 245-246; Quérard, 1833: 444; Larousse, 1873: 948), tantôt de l'italien (Barbier, 1874: 788; Laufer, 1971: 200). Pourtant, Quérard (1833: 444) signale que 'l'auteur paraît n'avoir fait autre chose que de mettre en meilleur français l'ouvrage intitulé: Histoire pitoyable du prince Erastus, fils de Dioclétien. Lyon et Anvers, 1568; Paris, 1587', publié sans nom d'auteur. Barbier (1874: 788) est du même avis et reprend les mots de Quérard.

$\mathrm{Au} \mathrm{XX}^{\mathrm{e}}$ siècle, Runte et al. (1984: 102) affirment sans preuves que l'Histoire du prince Erastus de Mailly est une "adaptation of a Spanish translation of the Italian Erasto". Plus récemment, Farrell et Andrachuk (Hurtado de la Vera, 1996: 20, 22-23 et 26) ont adhéré à cette même théorie selon laquelle Mailly aurait tiré son Histoire du prince Erastus de la Historia lastimera del principe Erasto d'Hurtado de la Vera, parue en 1572. En fait, comme nous avons découvert, cet ouvrage espagnol et l'Histoire pitoyable du prince Erastus anonyme sont deux traductions d'un même texte: I compassionevoli avvenimenti d'Erasto, dont la première édition daterait de $1542^{16}$ (Hurtado de la Vera, 1996: 22-23).

Néanmoins, si l'on se reporte à l'“Avertissement" de la première édition du texte de Mailly (1709c) on constate -tel que l'avaient signalé Quérard et Barbier-que le chevalier ne fit autre chose qu'adapter l'Histoire pitoyable du prince Erastus, fils de Diocletien, empereur de Rome (Lyon, 1564). L'auteur évoque les versions italienne et espagnole mais il souligne l'existence d'une version en "vieux François" qu'il aurait améliorée car, quand il emploie le verbe traduire, il faudrait plutôt comprendre réécrire ${ }^{17}$ :

14 Quadrio ajoute qu'un autre auteur anonyme composa une nouvelle intitulée Eraste (Paris, 1664), inspirée de ce roman mais de piètre valeur.

15 Farrell et Andrachuk (Hurtado de la Vera, 1996: 20) signalent qu'il y eut douze éditions de ce texte entre 1564 et 1616. Les catalogues de WorldCat et de la BnF nous ont permis de retracer jusqu'à quinze éditions de l'Histoire pitoyable du prince Erastus: Lyon, 1564, 1565, 1584 et 1585; Paris, 1565, 1566, 1570, 1572, 1579, 1584, 1587 et 1639; Anvers, 1568; Rouen, 1616 et 1646.

16 Selon Farrell et Andrachuk (Hurtado de la Vera, 1996: 15), les ouvrages provenant de la versio italica contiennent quatorze récits insérés: sept contés par les sages qui s'alternent avec six narrés par la marâtre et, enfin, celui d'Erastus. Or, les textes appartenant à la versio italica qui sont issus de I compassionevoli avvenimenti d'Erasto se caractérisent par l'absence de trois de ces contes -nommés Avis, Vidua et Puteus- et l'introduction de quatre autres, inconnus à toutes les autres versions, dont trois sont narrés par des philosophes et un par l'impératrice (Hurtado de la Vera, 1996: 18). Ces quatre nouvelles histoires sont Zelus, Corpus delicti, Caepulla et Puer adoptatus. En ce qui concerne les récits insérés dans l'ouvrage de Mailly -et donc dans l'anonyme français et dans celui d'Hurtado-, il s'agit de: Canis, Arbor, Medicus, Aper, Tentamina, Sapientes, Zelus, Gaza, Inclusa, Roma, Corpus delicti, Virgilius, Caepulla, Puer adoptatus, Vaticinium.

17 La réécriture est une pratique littéraire récurrente depuis l'Antiquité, dont les modalités et fonctions peuvent 
cette Histoire [...] a d'abord été écrite en Grec. On l'a [...] traduite en plusieurs Langues, \& particulierement en Italien, \& en Espagnol. Elle a même été mise en vieux François, mais d'un stile si diffus \& si barbare, que j'ay crû pour l'honneur de nôtre Nation, la devoir traduire dans un langage plus poli, \& plus digne de la beauté de son Original. La voicy donc dans le meilleur état que j'ay pû la mettre. (Mailly, 1709c: s. p.)

Dans le but d'éclaircir la véritable source de l'Histoire du prince Erastus, nous avons comparé quelques extraits du texte d'Hurtado de la Vera et de l'anonyme français ${ }^{18}$ avec la version du chevalier de Mailly. L'indice le plus important est que le cinquième chapitre de $I$ compassionevoli avvenimenti d'Erasto manque dans la traduction d'Hurtado, alors qu'il est présent dans l'ouvrage de Mailly. Cela prouverait que ce dernier ne provient pas du texte espagnol mais de l'anonyme français, où ce chapitre ne fait point défaut.

En ce qui concerne le style du roman, l'on remarque que Mailly fait usage de l'amplificatio là où son compatriote l'avait fait ou, du moins, là où l'auteur espagnol avait omis des passages de l'original italien que ces trois textes ont en commun. Voici un exemple: chez Hurtado de la Vera (1996: 122), l'histoire insérée dans le chapitre quatorze conclut sur les vers qu'écrit le personnage principal de celle-ci avant de se pendre, puis retourne immédiatement au premier niveau diégétique avec les mots que le philosophe Enoscopus adresse à Dioclétien. La fin de ce même chapitre dans l'anonyme français enchaîne ces vers avec la description de la pendaison du héros: "Et aiant parachevé d'escrire les vers que dessus, il ietta sa dague à bas \& poussant avec le pied vne scabelle sur laquelle il estoit monté, pour attacher au plancher le licol dont il se vouloit estrangler, il demeura pẽdu" (Histoire pitoyable, 1584: 172). Notre auteur introduit lui aussi la scène du suicide, qu'il reprend sans doute de la version française préalable puisqu'il dit: “Après avoir achevé d'écrire ces vers, il jetta sa dague à terre, \& poussa avec le pied une escabelle sur laquelle il étoit monté, afin d'attacher la corde, dont il vouloit s'étrangler, il s'y pendit" (Mailly, 1709c: 199).

Également, l'on observe que Mailly reste proche du texte de son compatriote quand Hurtado de la Vera emploie l'abbreviatio. Au début du dixième chapitre de l'anonyme français on peut lire:

Cependant neantmoins Dimurgus Philosophe, à qui touchoit de defendre le Prince Erastus, pour le second iour, de peur de surprinse, avoit à bien pourpensé tout ce qu'il auoit à dire à l'Empereur. Et ainsi estãt prest de plaider de bon matin, auant qu'on touchast rien au fait d'Erastus, il vint à la chambre de l'Empereur, où par fortune il le trouua desia sortant de sa chambre plustost que de coutume, pour les grandes facheries qui

être multiples. Il semble ici que Mailly voulait rendre plus accessible et agréable à lire un texte qu'il trouvait mal écrit mais dont il devait connaître l'importance. Pour un approfondissement du concept veuillez consulter La Seconde main d'Antoine Compagnon (Paris, Seuil, 1979), Palimpsestes, la littérature au second degré de Gérard Genette (Paris, Seuil, 1982) ou, sur le Grand Siècle en particulier, le numéro 186 (janvier-mars 1995) de la revue XVIIe siècle, sur La Réécriture au XVII siècle.

18 Puisque nous ignorons quelle édition de l'Histoire pitoyable du prince Erastus aurait pu lire Mailly, nous avons décidé de travailler avec l'édition de Paris 1584, qui se trouve en libre accès sur Gallica. 
le trauailloient \& en corps \& en esprit, ainsi qu'on peut presumer. (Histoire pitoyable, 1584: 71)

Mailly (1709c: 86-87), sans reprendre littéralement son antécédent anonyme, suit de près la progression de ce passage:

Comme c'étoit au Philosophe Dimurgus à parler le second jour pour la défense du Prince; il avoit étudié toute la nuit ce qu'il devoit dire à l'Empereur, qu'il trouva sortant de sa Chambre plûtôt que de coûtume, à cause du chagrin \& de la colere qu'il avoit contre le Prince son fils.

Par contre, Hurtado de la Vera (1996: 91), tout en introduisant une métaphore guerrière, reste bien plus succinct dans sa rédaction: "Dimiurgo, philósopho, mantenedor de la justa de aquel día, se puso en el campo con sus armas harto temprano, que fue bien menester por el madrugar d'el cansado Emperador de su atribulado pensamiento".

D'autre part, Farrell et Andrachuk (Hurtado de la Vera, 1996: 31) observent que l'écrivain espagnol tend à changer les termes employés par l'auteur de l'original italien pour leur conférer plus de force ou de transcendance. Par exemple, dans I compassionevoli avvenimenti d'Erasto, Aphrodisia, décidée à se venger d'Erastus pour l'avoir rejetée, appelle ainsi son mari: "Vieni adunque, e vindica me e te di tanta ingiuria"; la traduction espagnole dit: "¡Llega pues y véngame d'esta injuria hecha a la casa imperial, [...]!” (Hurtado de la Vera, 1996: 32). Par contre, les mots de Mailly (1709c: 61) -"Vengés-moy, vengés-vous de l'outrage que cet indigne nous a fait"- sont plus proches de l'original italien, qui lui était parvenu à travers l'anonyme français qui, lui, dit: "Venez donc, \& me vengez, \& vous aussi du grand outrage que ce malheureux [...] m'a voulu faire" (Histoire pitoyable..., 1584: 53).

Quant aux différences du texte de Mailly par rapport à la traduction française du XVI ${ }^{\mathrm{e}}$ siècle qu'il réécrit, on peut noter qu'elles relèvent parfois de l'abbreviatio. Mailly supprime, par exemple, la description du cadre spatio-temporel donné au premier chapitre de l'Histoire pitoyable... (1584: 5) et selon lequel cette aventure aurait eu lieu "l'An mil quarãte, prins à la fondation de Romme", du temps où "Diocletien Empereur, Prince fort sage \& fort puissant, estoit en regne en la superbe \& triomphante Rõme, enuiron 283. ans apres la mort de nostre Seigneur Iesus Christ".

Toutefois, le procédé le plus habituel dans cette œuvre est l'amplificatio. Il s'agit de développements qui cherchent souvent à donner un tour de galanterie au texte et qui ont été pensés pour plaire au nouveau public. Dans ce sens, Mailly (1709c: 13) énumère des divertissements à la mode au XVII ${ }^{\mathrm{e}}$ siècle et qui auraient eu lieu lors des noces de Dioclétien et Aphrodisia: "des Fêtes publiques, des bals, des mascarades, des tournois, des courses de bagues \& de têtes, des combats de barrieres \& de chariots". Également, la description du prince Erastus rapportée à l'impératrice est typiquement romanesque et constitue un nouveau clin d'œil au lecteur contemporain: 
on luy dit qu'il étoit d'une mine \& d'un port admirable; que sa taille étoit bien prise, \& au dessus de l'ordinaire; que ses cheveux étoient blonds \& annelez, \& luy tomboient jusqu'à la ceinture; que jamais on ne vit de plus beaux yeux ny de plus belles dents; que tous les traits de son visage faisoient un assemblage merveilleux; que ses couleurs étoient d'un blanc le plus vif au monde, mêlé avec un incarnat surprenant; \& qu'enfin son corps étoit si bien proportionné, \& d'un air si beau \& si majestueux, qu'on ne pouvoit le voir sans l'admirer. (Mailly, 1709c: 14)

Il n'est pas rare non plus que le chevalier étoffe des critiques déjà développées par l'auteur de l'Histoire pitoyable du prince Erastus. Tel est le cas dans le discours que Merlin adresse à un roi de Londres, abusé par ses conseillers, et que l'on a l'impression que Mailly (1709c: 162-163) adresse implicitement à Louis XIV:

Ainsi demandés-leur pardon, \& faites mourir ces sangsuës publiques, ces monstres parcides [sic] de l'Etat; qui comme les enflures de la peste ne grossissent que de la maigreur des autres membres, qui ne vivent que de l'agonie des Peuples, ne boivent que leurs larmes, \& dont l'insatiable ardeur tirant tous les nerfs du corps politique, luy cause des convulsions mortelles, \& une espéce de paralysie morale.

De même, Mailly (1709c: 251) semble renforcer la critique de la fausse vertu de certaines femmes: “Elles nous assassinent continuellement d'un honneur \& d'une vertu qu'elles n’ont qu'en apparence". Nonobstant, érigé en champion du Beau Sexe grâce à ses ouvrages adressés aux Dames, il essaye de nuancer cette attaque en faisant mention et louange des femmes qui sont véritablement honnêtes: "celles qui sont effectivement vertueuses n'en tirent point vanité; \& leur conduite est moins suspecte que la manière d'agir des autres" (Mailly, 1709c: 251-252).

Si le chevalier modifie parfois le contenu ou le ton de certains passages pour rapprocher l'ouvrage de son public, il arrive aussi qu'il change certaines expressions pour embellir et raffiner son texte. Par exemple, notre homme de lettres transforme une phrase anodine -“comme vray amant qu'il estoit, ne trouuant aucune chose impossible, apres auoir longuemẽt discouru en son cerueau sur ce qu'il auoit a faire [...]" (Histoire pitoyable..., 1584: 212)- en une formule à l'air proverbial, qui s'insère parfaitement dans un contexte où Fabio cherche le moyen de pénétrer chez sa maîtresse: “comme il n'y a pas de maison si bien fermée qu'un Chat \& un Amant n'y puissent entrer [...]” (Mailly, 1709c: 244).

\section{De Il Peregrinaggio di tre giovanni figlivoli del re di Serendippo (1557) de Christoforo} Armeno au Voyage et les avantures des trois princes de Sarendip (1719)

Les épisodes narrés dans l'ouvrage de Mailly furent, d'après le sous-titre, "traduits du Persan", mais aucune référence bibliographique au texte source n'est faite dans le paratexte ${ }^{19}$.

19 Cela explique pourquoi les bibliographes qui ont répertorié l'ouvrage de Mailly ont presque tous indiqué “tra- 
L'œuvre à l'origine de cette traduction est pourtant italienne; elle s'intitule Il Peregrinaggio di tre giovanni figlivoli del re di Serendippo, per opra di M. Christoforo Armeno, dalla persiana nell'italiana lingua trapportato, dont la première édition semble avoir été publiée à Venise, chez Michele Tramezzino, en 1557 (Barbier, 1874: 1088 et Robert, 2002: 59).

L'identité de Christoforo Armeno ou Christophe l'Arménien reste obscure et pourrait même suggérer un nom de plume. Or, Merton et Barber (2004: 15) rapportent que, selon Joseph Schick $^{20}$, ce Christoforo était un Arménien qui, vers le milieu du XVI ${ }^{\mathrm{e}}$ siècle, aurait passé trois ans à Venise, où il aurait écrit son Peregrinaggio, s'inspirant du Hast Bihist d'Amir Khosrau, un auteur persan qui vécut entre le XIII ${ }^{\mathrm{e}}$ et le XIVe siècle. Boyle (2000: II) affirme que le livre contient des fables indiennes du Panchatantra, du Talmud juif et du folklore coréen, ukrainien, serbe et croate. Néanmoins, il n'y a pas de consensus entre les érudits à propos des sources d'Armeno. "Faute de connaître le texte oriental, s'il en existe un, dont s'inspire le narrateur italien quand il prétend traduire du persan son Voyage des trois jeunes fils du roi de Sérendip, il est impossible d'analyser rigoureusement ce dernier récit", regrette Robert (2002: 67). D'autre part, il faut également considérer l'hypothèse selon laquelle l'Arménien eût recours à des sources folkloriques orales venues d'Orient ou, tout simplement, au prétexte littéraire de la fausse traduction ou du faux texte ancien pour rendre plus attirant son ouvrage. Boyle (2000: I) va jusqu'à se demander si l'auteur du Peregrinaggio ne serait pas l'éditeur de l'ouvrage, Michele Tramezzino.

Quoi qu'il en fût, Merton et Barber (2004: 15) signalent que les nombreuses éditions qui se firent du Peregrinaggio prouvent qu'il fut très apprécié: “There is considerable evidence for the popularity of Christoforo's work. Four editions in Italian appeared within less than a century: in 1584, 1611 [...], 1622, and 1628”. De même, les diverses traductions que l'ouvrage inspira témoignent d'un succès éditorial considérable:

It was translated by Johann Wetzel into German and published in Basel in 1583, and this edition was republished in 1599, and again, in a reworked form, in 1630. In French, there appeared one translation by François Beroalde in 1610, a very free translation by Simon Gueulette ${ }^{21}$ in 1712 (which Voltaire used for Zadig), and a more accurate one by

duit du Persan par le Chevalier de Mailly". C'est le cas de Weiss (1820b: 246), de Quérard (1833: 444), de J.V. (1860: 888), de Larousse (1873: 948) et, actuellement, du catalogue de la Bibliothèque Sainte Geneviève.

20 C'est le dernier critique à s'être prononcé sur la question, dans Die Scharfsinnsproben, vol. 4, partie 1 du Corpus Hamleticum (Leipzig, Harrassowitz, 1934) (Merton et Barber, 2004: 15).

21 Raymonde Robert (2002: 60 et 68) assure que cette adaptation de Gueullette, Les Soirées bretonnes, est "un continuel démarquage du récit de l'Arménien" et relève davantage du registre merveilleux car, "[d]e la favorite ambitieuse et rusée du texte italien, il ne reste pas grand-chose dans la malheureuse, innocente et noble victime du conte de fées". En outre, les déguisements endossés par les héros à un certain moment de l'histoire "se situent davantage dans la tradition des spectacles contemporains de Gueullette, opéra et comédie, genres avec lesquels les contes de fées du XVIII ${ }^{\mathrm{e}}$ siècle entretiennent des rapports certains" (Robert, 2002: 69). Bahier-Porte (2007: 138) affirme que Les Soirées bretonnes ont deux sources principales: d'un côté, "Le Voyage des trois fils du roi de Sarendip que Christoforo Armeno dit traduire du persan en 1557" et, d'un autre côté, "L'Histoire véritable ou Le voyage des princes fortunés (1610) de Beroalde de Verville qui se fonde également en partie sur le recueil d'Armeno". 
de Mailly in 1719. From de Mailly's translation three further translations were made, into English in 1722 (The Travels [...], which Walpole read), into German in 1723, and into Dutch in 1766. (Merton et Barber, 2004: 15-16)

En ce qui concerne la fidélité de la traduction de Mailly, les avis sont partagés, ce qui invite à croire que les critiques ne se reportent pas souvent aux ouvrages originaux puisque, comme nous allons le voir par la suite, le chevalier demeure parfois très attaché au texte source mais, d'autres fois, il s'en éloigne considérablement. En 1788, l'éditeur du tome vingt-cinquième des Voyages Imaginaires, où est insérée la version de Mailly du Voyage et les avantures des trois princes de Sarendip, pense que le lecteur "trouvera ici avec plaisir une traduction exacte" du texte d'Armeno (Mailly, 1788: xi). Par contre, plus récemment, Robert (2002: 61) affirme que ni Gueullette ni Mailly ne retiennent l'épisode où, lors d'un concours d'adresse au tir à l'arc, l'on transforme une licorne mâle en femelle et vice-versa. Cette remarque est fausse, du moins pour le texte de Mailly (2011: 66) car, non seulement il n'omet pas ce passage mais il le traduit très scrupuleusement. Robert (2002: 61) poursuit sa démonstration et constate que "Gueullette insérera toutes les autres anecdotes qui viennent d'être citées $^{22}$ [...]. Quant au chevalier, il se contente de reprendre, comme Gueullette d'ailleurs, l'intégralité d'une nouvelle insérée dans le récit principal", celle de la métempsycose. En effet, Mailly traduit cette histoire-ci, mais il ne néglige pas d'insérer dans son texte les autres épisodes que Robert semble lui contester. Au début du XXI siècle également, Boyle (2000: II) signale que Mailly "added other stories, so this version does not accurately reflect Tramezzino's work".

Toutefois, il a fallu attendre les années 2010 pour qu'une étude approfondie du Voyage et les aventures des trois princes Sarendip de Mailly soit faite. Dans son analyse, Aude Volpilhac (Mailly, 2011) constate que l'ouvrage du chevalier débute, en effet, comme une traduction fidèle, mais que l'auteur se dégage petit à petit du texte source en introduisant des éléments nouveaux et finit par changer la fin du conte-cadre, tout en ajoutant une dernière nouvelle autonome. D'ailleurs, l'amplificatio de Mailly peut être observée rien qu'en comparant la longueur de son Voyage et celle du Peregrinaggio: au même format, celui en italien s'étend sur cent soixante-six pages, alors que celui en français en prend trois cent quatrevingt-une. En effet, l'ouvrage d'Armeno réunit sept nouvelles, tandis que celui de Mailly en présente neuf et, dès la quatrième, le chevalier introduit de plus en plus de modifications. Volpilhac (Mailly, 2011: 194) déclare que l'ouvrage de notre homme de lettres est "le fruit d'une combinaison complexe d'emprunts, de réminiscences, de réécritures et d'inventions", tirés aussi bien de textes anciens que récents. En tout cas, il est important de signaler, selon

22 À savoir, l'épisode du chameau, celui du repas où l'on sert de l'agneau qui a un goût de chien et du vin qui a un goût de mort, et celui de la tentative du vizir d'empoisonner le roi. Puis, les anecdotes concernant des épreuves de tir à l'arc: le tireur qui traverse d'une même flèche la patte et l'oreille d'un cerf, le défi pour décocher, dans le noir, trois flèches dans un bassin et, enfin, celui de transformer, à l'aide d'un arc et de flèches, une licorne mâle en femelle et inversement (Robert, 2002: 60-61). 
Volpilhac (Mailly, 2011: 195-198), que l'atmosphère orientale originale s'estompe au fur et à mesure que l'auteur insère ses propres récits, qui relèvent surtout de la nouvelle galante qu'il privilégia dans l'ensemble de sa production littéraire.

À cette occasion, comme il en a l'habitude, le chevalier ne manque pas de reprendre certains passages, voire des anecdotes en entier, dans de nouveaux ouvrages. Mailly avait déjà utilisé l'épisode de la métempsycose qui paraîtrait en 1719 dans Le Voyage et les avantures des trois princes de Sarendip, dans son conte intitulé "Le Bienfaisant ou Quiribirini", compris dans Les Illustres Fées (1698). Puis, nous retrouvons dans Avantures et lettres galantes... (Mailly, 1718: 9-11) et dans L'Eloge de la chasse... (Mailly, 1723: 25-28), 1'histoire -insérée dans la huitième nouvelle du Voyage et les avantures des trois princes de Sarendip - de la bergère belle et sage qui donne à boire à un roi qui s'éprend immédiatement d'elle. Enfin, L'Eloge de la chasse... (Mailly, 1723: 107-147) présente dans "De la Chasse des Licornes" un démarquage de la première nouvelle de l'œuvre d'Armeno.

Cependant, le conte-cadre de l'Arménien et les principaux récits insérés de celui-ci sont respectés par le chevalier de Mailly dans sa traduction. À la base de ces historiettes orientales il y a généralement des énigmes.

Tales of detection of this kind -tales of Scharfsinnsproben, as German scholars have called them- had their origin in antiquity in the Far East, in India and China, and in the Semitic countries of the Near East. They have many themes : the discovery of paternity and bastardy, the distribution of an inheritance, and, finally, the description either of an unseen object or of the provenance of a known object from various clues and traces. [...] Similar stories can be found in the Babylonian Talmud, in the Jewish Midrasch Ekāh, and much later, in the eighteenth century, in the Arabian Nights story of the "Sultan of Yemen and His Three Sons". René Bastet, in his Contes Populaires d'Afrique, recounts yet another similar story [Paris, Guilmoto, 1903]. (Merton et Barber, 2004: 14-15)

L'on considère généralement qu'à la base du modèle occidental du déchiffreur d'énigmes se trouvent certains récits de la Bible (par exemple celui où Daniel démasque les prêtres du dieu Bêl), des mythes et des textes de l'Antiquité -comme Edipe-Roi de Sophocle- et quelques ouvrages du XVIII' ${ }^{\mathrm{e}}$ siècle (Reuter, 2009: 14). Dans Il Peregrinaggio di tre giovanni figlivoli del re di Serendippo et, donc, dans Le Voyage et les avantures des trois princes de Sarendip, l'aventure du chameau, celle de l'agneau et du vin au repas, ainsi que celle de la découverte de la trahison du vizir sont de ce genre. L'épisode du chameau est devenu particulièrement célèbre et a fait couler beaucoup d'encre depuis le XVIII' siècle. “Dans l'Année littéraire de 1767 (t. I, 145), Fréron accusa Voltaire d'avoir pris dans cet ouvrage le chapitre du roman de Zadig, intitulé du Chien et du Cheval" (Chaudon et Delandine, 1810: 514) ${ }^{23}$. Plus tard, Loiseleur-Deslongchamps (1838: 56) affirmait que Voltaire tira son

23 Après eux, Weiss (1820b: 246), Quérard (1833: 444), J. V. (1860: 888), Larousse (1873: 948) ou Barbier (1879: 1088) se sont fait l'écho de cette remarque. 
conte des Soirées bretonnes de Gueullette. “Ascoli dans son édition critique de Zadig (1929: II, 31) étudie les sources de l'épisode [...] [et] conclut que le récit du chevalier de Mailly est moins voisin de celui de Voltaire que ceux de d'Herbelot (Bibliothèque Orientale, 1697) et de Gueullette" (Gueullette, 1977: 96, n. 1). Au XX siècle, Carolyn Wells (1913: chap. VIII, $\S 1)$ signale la ressemblance entre le texte de Voltaire et celui de Mailly, qu'elle dit tiré du conte des Mille et Une Nuits "Le Sultan d'Yémen et ses trois fils"; elle ne fait donc aucune mention du Peregrinaggio d'Armeno.

L'histoire du sultan d'Yémen, qui ne figure que dans le manuscrit Wortley-Montague des Mille et Une Nuits, correspond au conte-type AT 655 A, The Strayed Camel and the Clever Deductions, et apparaît déjà dans le Talmud et le Midrash hébraïques, ainsi que, dans la littérature du $\mathrm{X}^{\mathrm{e}}$ siècle en langue arabe, dans les travaux de l'historien Al-Tabarî et dans le Murûj al-dhahab d'Al-Mas'ûdî's (Marzolph et al, 2004: 403). Puis, en Europe, l'histoire du chameau "appears in Italian literature for the first time in the writings of one of Boccaccio's students, Giovanni Sercambi (1344-1424), as a tale called 'De Sapientia'” (Merton et Barber, 2004: 15). Cet épisode a été repris autant de fois qu'il ne serait donc pas correct de dire qu'il a été plagié par Voltaire, d'où que l'accusation de Fréron soit tout à fait injuste ${ }^{24}$ (Merton et Barber, 2004: 19, n. 45). En fait, pour certains critiques comme Alfredo Arias, l'auteur des Lumières ne fit pas une copie mais une recréation du sujet, donnant ainsi une imitatio au sens classique du terme (Chandler, 2005: 14).

Ce fameux épisode et, plus généralement, l'histoire des trois princes de Sarendip connurent une fortune extraordinaire à partir du XVIII ${ }^{\mathrm{e}}$ siècle dans des pays de langue non-romane. Déjà au Siècle des Lumières, Horace Walpole aurait fait la lecture du texte de Mailly, ce qui l'aurait mené à forger le terme de serendipity -ou sérendipité, en français- (Boyle, 2000: II et Mailly, 2011: 203-223). Au XIX ${ }^{\mathrm{e}}$ siècle, Wilhelm Hauff, célèbre conteur allemand, reproduisit presque à l'identique la technique déductive du héros de Zadig dans son Märchen-Almanach für Söhne und Töchter gebildeter Stände auf das Jahr 1827 [Almanach de contes de fées pour les fils et les filles de la bonne société de l'année 1827] (Chandler, 2005: 13). Enfin, Une Étude en rouge d'Arthur Conan Doyle rendit populaire, dès 1887 jusqu'à nos jours, le système d'analyse de Zadig, à travers le personnage de Sherlock Holmes (Chandler, 2005: 13).

\section{Conclusions}

Dans un contexte où le français cherche à se parfaire et à s'imposer comme langue d'érudition, la traduction se révèle un outil indispensable, tel que nous l'avons souligné dans la deuxième section de cet article. D'après nos recherches, le chevalier de Mailly traduisit deux ouvrages de l'italien -La Vie d'Adam (1695), d'un texte de Loredano, et Le Voyage et

24 Et malintentionnée aussi car n'oublions pas que ces deux auteurs se professaient une haine implacable. 
les avantures des trois princes de Sarendip (1719) à partir de l'œuvre d'un certain Christoforo Armeno-, ainsi que quelques paragraphes d'un roman espagnol de Vélez de Guevara, dans Diverses Avantures de France et d'Espagne (1707). Quant à l'Histoire du prince Erastus (1709), cet ouvrage ne serait pas une traduction, mais une réécriture d'un anonyme français du $\mathrm{XVI}^{\mathrm{e}}$ siècle dont la langue et le style furent jugés surannés et peu élégants par Mailly. Deux décennies se sont écoulées entre la première et la dernière traduction faites par notre auteur. Nous avons constaté dans les traductions du chevalier que celui-ci est, au fur et à mesure que le temps passe et qu'il devient un écrivain plus expérimenté, de moins en moins fidèle au texte source. Il se produit donc une évolution où le traducteur devient adaptateur - puisqu'il se permet d'introduire des détails, d'étoffer des descriptions ou des histoires insérées- et, finalement, auteur à part entière -en créant de nouvelles intrigues à partir des originaux qui l'inspirent-.

\section{Références bibliographiques}

Argaud, Evelyne. 2009. "Les enjeux des représentations des langues savantes et vulgaires en France et en Europe aux XVI ${ }^{\mathrm{e}}$ et XVII ${ }^{\mathrm{e}}$ siècles. Affirmer des prééminences et construire une hiérarchisation" in Documents pour l'histoire du français langue étrangère ou seconde, no 43, 1-12: <https://journals.openedition. org/dhfles/815> [22/05/2020].

BArbier, Antoine-Alexandre. 1874. Dictionnaire des ouvrages anonymes. Tome II. Paris, Daffis, 3ème éd.

Chaudon, Louis-Mayeul \& Antoine-François Delandine. 1810. Dictionnaire Universel, Historique, Critique et Bibliographique. Tome X. Paris, Prudhomme fils.

Donneau De Visé, Jean (sous la dir. de). 1695a. "Vie d'Adam" in Mercure galant, octobre 1695. Paris, Michel Brunet, 308-310.

Donneau De Visé, Jean (sous la dir. de). 1695b. "Lettre sur le Livre de la vie d'Adam" in Mercure galant, décembre 1695, 73-86. Paris, Michel Brunet.

Donneau De Visé, Jean (sous la direction de). 1709. "Livres nouveaux" in Mercure galant, décembre 1709. Paris, Michel Brunet, 187-198.

Dotoli, Giovanni. 2004. Littérature et société en France au XVII siècle. Vol. IV. Paris, Presses de l'Université de Paris-Sorbonne (coll. "Biblioteca della Ricerca”, II. Cultura straniera, $n^{0} 131$ ).

FURETIÈRe, Antoine. 1658. Novvelle allegorique ou Histoire des derniers trovbles arrivez au Royavme d'Eloqvence. Paris, Guillaume de Luyne.

Gueullette, Jean-Émile. 1977. Un Magistrat du XVIII siècle Ami des Lettres, du Théâtre et des Plaisirs: Thomas-Simon Gueullette. Genève, Slatkine Reprints.

Histoire pitoyable du prince Erastus, fils de Diocletien, Empereur de Rome. Ou sont contenus plusieurs beaux exemples \& notables discours, non moins plaisans \& recreatifs, qu'vtiles \& profitables. Reuеuё, corrigee, \& enrichie de plusieurs portraits \& figures, non encore par cy 
deuant imprimez. Traduicte d'Italien en François. 1584. Paris, Hiersome [sic] de Marnef \& la veufue de Guillaume Cauellat.

Hurtado de la Vera, Pedro. 1996. Historia lastimera d'el príncipe Erasto. Édition critique, notes et étude préliminaire d'Anthony J. Farrell et Gregory Peter Andrachuk. Salamanque, Editions de l'Université de Salamanque (coll. Textos recuperados $n^{\circ}$ XIV).

LANCELOT, Claude. 1660. Novvelle methode povr apprendre facilement et en pev de temps la langue italienne. Paris, Pierre Le Petit.

Larousse, Pierre. 1873. Grand dictionnaire universel du XIX ${ }^{\mathrm{e}}$ siècle. Tome 10. Paris, Administration du grand Dictionnaire universel.

LAUfER, Roger. 1971. Lesage ou le métier de romancier. Paris, Gallimard et NRF (coll. Bibliothèque des idées).

Loiseleur-DeslongChamps, Auguste. 1838. Essai historique sur les contes orientaux et sur les Mille et Une Nuits. Paris, Auguste Desrez.

Loredano, Giovanni Francesco. 1666. L'Adamo di Gio. Francesco Loredano, Nobile Veneto. Venise, Il Valvasense, ottava impressione.

Mailly, Louis de. 1695. La Vie d'Adam. Paris, Edme Couterot.

MaIlly, Louis (de). 1709. Histoire du prince Erastus, fils de l'Empereur Diocletien. Paris, Pierre Mergé.

MaIlly, Louis de. 1718. Avantures et lettres galantes, avec la Promenade des Tuilleries. Amsterdam, Etienne Lucas.

MaIlly, Louis de. 1719. Le Voyage et les avantures des princes de Sarendip. Traduit du Persan. Paris, Pierre Prault.

MaILly, Louis de. 1723. Éloge de la chasse, avec plusieurs aventures surprenantes et agréables qui y sont arrivées. Paris, Jean-Luc Nyon.

MaILly, Louis de. 1788. "Le Voyage \& les Aventures des trois Princes de Sarendip; traduits du Persan" in Voyages imaginaires, songes, visions, et romans cabalistiques. Tome vingt-cinquième. Amsterdam et Paris, Rue et Hôtel Serpente.

MaILly, Louis de. 2011. Aventures des trois princes de Serendip. Suivi de Voyage en Sérendipité par Aude Volpilhac, Dominique Goy-Blanquet et Marie Anne Paveau. Vincennes, Thierry Marchaisse.

Merton, Robert K. \& Elinor Barber. 2004. The Travels and Adventures of Serendipity. A Study in Sociological Semantics and the Sociology of Science. Princeton et Oxford, Princeton University Press.

QuAdrio, Francesco Saverio. 1749. Della storia, e delle ragione d'ogni poesia. Dove le cose all'Epica appartenenti sono comprese. Volume quarto. Milan, Francesco Agnelli.

QuÉRARD, Joseph-Marie. 1833. La France littéraire, ou Dictionnaire bibliographique des savants, historiens et gens de lettres de la France, ainsi que des littérateurs étrangers qui ont 
écrit en français, plus particulièrement pendant les XVIII et XIX' ${ }^{\mathrm{e}}$ siècles [...]. Tome 5. Paris, Firmin Didot frères.

ROBERT, Raymonde. 2002. Le conte de fées littéraire en France de la fin du XVII à la fin du XVIII siècle. Supplément bibliographique 1980-2000 établi par Nadine Jasmin avec la collaboration de Claire Debru. Paris, Honoré Champion (coll. Lumière classique no 40).

Runte, Hans R., J. Keith Wikeley \& Anthony J., Farrell. 1984. The Seven Sages of Rome and The Book of Sindbad: An Analytical Bibliography. With the collaboration of the Society of the Seven Sages. New York, Garland.

Runte, Hans R. 2014. Portal, Society of the Seven Sages: <http://dalspace.library.dal.ca / handle/10222/49107> [12/05/2020].

STORER, Mary Elizabeth. 2011. Un épisode littéraire de la fin du XVII siècle: la mode des contes de fées (1685-1700). Genève, Slatkine Reprints.

Vega, Miguel Ángel. 1994. Textos clásicos de teoría de la traducción. Madrid, Cátedra.

Vélez De Guevara, Luis. 1641. El Diablo cojuelo. Madrid, Alonso Pérez.

WeIss, Charles. 1820. Biographie universelle ancienne et moderne. Tome 26. Paris, Michaud. 
